\title{
Unknown Values Estimation in Incomplete Fuzzy Soft and Interval Valued Fuzzy Soft Matrices
}

\author{
Seema Singh ${ }^{1}$, D. S. Hooda ${ }^{2 *}$, S. C. Malik ${ }^{3}$ \\ ${ }^{1,3}$ Department of Statistics, M. D. University \\ Rohtak, India \\ ${ }^{2}$ Honorary Professor of Mathematics, G.J. University of Science and Technology \\ Hisar, India \\ *Corresponding author's email: $d s_{-}$hooda [AT] rediffmail.com
}

\begin{abstract}
In day to day problems, incomplete information due to unknown values in data is the cause of the loss of information which leads to uncertainly, ambiguity and vagueness. There are many reasons for unknown values in data, like errors in data collection, lack of data information, inappropriate technique and illegibility of data which cause incompleteness in data. Thus, to estimate the unknown values in data of various information systems is an important area of research. In this communication, the definitions of incomplete Fuzzy soft and interval-valued fuzzy soft matrices are given with application in numerical problems. An algorithm is proposed for unknown values estimation in an incomplete fuzzy soft matrix and applied in solving a numerical problem. An application of incomplete fuzzy soft matrix after inserting the unknown values in dimension reduction is studied. The unknown values in the incomplete intervalvalued fuzzy soft matrix are also estimated and applied in multi-criteria decision-making method.
\end{abstract}

Keywords and Phrases---- Fuzzy soft set, Interval-valued Fuzzy soft set, Estimation, Fuzzy Soft Matrices and Dimension Reduction

\section{INTRODUCTION}

Zadeh [27] defined a fuzzy set to describe the uncertainty due to ambiguity and impreciseness in the environment of vagueness and applied to solve the complex real life problems. Subsequently, a number of generalizations of the fuzzy set were developed applied in many fields fo science and engineering. In view of the fact that fuzzy set has inadequacy of parameterization, Molodtsov [19] evolved soft set theory and that is considered a better theory in comparison to any other theory, like fuzzy set, rough set, and statistical approach, to deal with parametric data.

Theoretical as well as applied research in soft set theory has attracted the attention of many scientists from different fields. Maji et al. [21] applied soft set theory in decision-making problems. Later on, their work was improved by Chen et al. [1], Kong et al. [14], and Ma et al.[23] sequentially. Besides these developments many other researchers, namely Celik and Yamak [4], Jun et al. [12], Kalaichelvi and Malini [17], Kalayathankal and Singh [16], Tanay and Kandemir [25] used soft set hybridization with fuzzy set theory for handling the uncertainty in vagueness of data related to daily life.

The theory of soft set was extended by Maji et al. [20,22] to fuzzy soft set theory. A computational tool based on a fuzzy soft set was introduced by Kong et al. [15]. That improved the traditional decision-making process and proved more convenient in case attributes were changed across the decision process. The theory of soft and fuzzy soft sets has proved useful in many fields such as forecasting, role mining, data analysis, simulation, and evaluation of sound quality.

There is a very convenient representation of soft and fuzzy soft sets in the form of Matrices. The advantage of representing a fuzzy soft set in the matrix form is that the data is easily stored and interpreted in a computer. Kandasamy et al. [13] initially introduced the concept of a fuzzy matrix. Cayman and Enginoglo [2] defined a soft matrix and multiplication of soft matrices. They further used the multiplication of soft matrices to describe a soft max-min decision-making method. Hooda and Jain [8] defined and characterized the information measures on fuzzy matrices and described their binary relations. Fuzzy soft matrix and its application in decision-making problems were introduced and studied by Cayman et al. [3]. 
It is easy to handle matrices when complete information is given, but the problem arises when some of the data values are missing due to mishandling, inaccurate measurement, or inappropriate tools for collecting information, etc. This leads to more uncertainty and ambiguity. Missing data occurs almost in all scientific fields, even in well-designed and controlled studies. Due to missing data, the statistical power of the study is reduced that leads to biased estimation and invalid conclusion. Thus, it becomes the utmost essential to estimate the unknown values in data instead of ignoring them.

A data analysis approach for soft sets with incomplete information was described by Zou and Xiao[28] . Their method is based on a weighted average of all possible choice values of the objects. The weight of each possible choice value from the probability of belonging of an object with a parameter is calculated. This method to compute the summation of all parameters' values for each object has a drawback as the unknown entries remain unknown. They also described an average probability method to predict the missing data in a fuzzy soft set. Actually, that method is based on the probability of an object belonging to a parameter, which is considered as the degree of belongingness of the object to that parameter.

Deng and Wang [5] defined an object parameter approach to estimate the missing values in the incomplete fuzzy soft set. In that technique, complete information from the relationship among various objects of a certain parameter and among the values of different parameters about a concerned object was considered. The new concept of complete distance between two objects and relative dominance degree between two parameters was introduced to explore the unknown information in a more precise and reasonable way.

Later on, Dong and Xiao[6] defined Dempster-Shafer's fuzzy soft set by combining Dempster-Shafer theory and fuzzy soft set. A decision-making algorithm for the incomplete fuzzy soft set was also described. A data filling approach using the relations between parameters and the association degree to measure the relation was explained by Qin et al.[24]. However, Khan et al. [18] also defined another data filling approach for unknown values estimation in soft sets. They mainly focussed on the reliability of association between parameters instead of probabilities.

Recently, Hooda and Barak [10] described an algorithm and applied in the estimation of the missing data in the design of experiments and contingency table. They have also studied the estimation of missing values in fuzzy matrices and extended application to interval-valued fuzzy matrices (refer to Hooda and Barak [11]). An algorithmic approach for predicting unknown information in incomplete fuzzy soft and interval-valued fuzzy soft sets was also given by Surjit et al. [7]. Wang et al. [26] also studied incomplete fuzzy soft set and its application to decision making.

In the present communication, a data filling approach to estimate unknown values in fuzzy soft and interval-valued fuzzy soft matrices is described and its application in numerical problems is illustrated. In section 2, some basic concepts used in the development of the paper were defined with examples. In section 3, an algorithm for unknown values estimation for a fuzzy soft matrix is proposed and illustrated with an example. In section 4, the application of fuzzy soft matrix in data dimension reduction is explained. An algorithm is proposed in section 5 for estimation of unknown values in an incomplete interval-valued fuzzy soft matrix and solved a numerical problem for illustration. A decision making method is also described by using of interval-valued fuzzy soft matrix in section 6. Discussion and conclusion are given in section 7 and the references are enumerated at the end of the paper.

\section{PRELIMINARIES}

In this section some basic concept which are used further for the development of the paper are defined.

\section{Definition 2.1[9]: Fuzzy Set}

Let $\mathrm{X}$ is a non-empty set of universe, a function $\mu_{A}(x): X \rightarrow[0,1]$ defines a fuzzy set $\mathrm{A}$ on $\mathrm{X}$ and usually written as

$$
A=\left\{\left(x_{i}, \mu_{A}\left(x_{i}\right)\right): \mu_{A}\left(x_{i}\right) \in[0,1] ; \forall x_{i} \in X\right\}, \text { where }
$$

$\mu_{A}\left(x_{i}\right)$ is called membership function which satisfies the following conditions:

$$
\mu_{A}\left(x_{i}\right)=\left\{\begin{array}{rr}
0, & \text { if } x_{i} \notin A \text { and there is no ambiguity } \\
1, & \text { if } x_{i} \in A \text { and there is no ambiguity } \\
0.5, & \text { there is max ambiguity whether } x_{i} \in A \text { or } x_{i} \notin A
\end{array}\right.
$$

\section{Definition 2.2[21]: Soft Set}

Let us consider ' $U$ ' as the universal set and $T$ be the parametric set, then the pair $(\pi, T)$ is defined as soft set over $U$ iff $\pi$ is a function of $\mathrm{T}$ to the power set of $\mathrm{U}$ i.e. 


$$
\pi: T \rightarrow P(U)
$$

Example2.2. Let $U=\left(t_{1}, t_{2}, t_{3}, t_{4}\right)$ be the set of four televisions and ' $E$ ' be the parametric set namely, $E=$ $\left(e_{1}(\right.$ clear screen $), e_{2}($ cheap $), e_{3}(\operatorname{good}$ body $\left.)\right)$. Then, the soft sets $(\pi, E)$ over $\mathrm{U}$ is given by

$$
(\pi, \mathrm{E})=\left\{F\left(e_{1}\right)=\left(t_{1}, t_{4}\right), F\left(e_{2}\right)=\left(t_{2}, t_{3}\right), F\left(e_{3}\right)=\left(t_{1}, t_{3}\right)\right\}
$$

Table 1: Tabular Representation of Soft Set

\begin{tabular}{|c|c|c|c|}
\hline$U / E$ & $e_{1}$ & $e_{2}$ & $e_{3}$ \\
\hline$t_{1}$ & 1 & 0 & 1 \\
\hline$t_{2}$ & 0 & 1 & 0 \\
\hline$t_{3}$ & 0 & 1 & 1 \\
\hline$t_{4}$ & 1 & 0 & 0 \\
\hline
\end{tabular}

\section{Definition 2.3[7]: Fuzzy Soft Set}

Let $X$ is a set of universe under discussion; $E$ is the set of parameters and $A \subseteq E$. Let $F(X)$ be the set of all fuzzy subsets of $\mathrm{X}$, then the pair $(\mathrm{F}, \mathrm{A})$ is called fuzzy soft set of $\mathrm{X}$, where $\mathrm{F}$ is a mapping from $\mathrm{A}$ to $\mathrm{F}(\mathrm{X})$.

Example2.3. Let us consider $\mathrm{C}=\left\{c_{1}, c_{2}, c_{3}\right\}$ be the set of three courses and $\mathrm{E}=\left\{e_{1}=\right.$ job oriented, $e_{2}=$ affilated, $e_{3}=$ cheaper $\}$ be the set of parameters and let $\mathrm{B}=\left\{e_{1}, e_{2}\right\} \subset E$, then

$$
(\mathrm{F}, \mathrm{B})=\left\{\begin{array}{l}
F\left(e_{1}\right)=\left\{c_{1} / 0.5, c_{2} / 0.9, c_{3} / 0.7\right. \\
F\left(e_{2}\right)=\left\{c_{1} / 0.6, c_{2} / 0.8, c_{3} / 0.4\right.
\end{array}\right.
$$

$(\mathrm{F}, \mathrm{B})$ is a fuzzy soft set over $\mathrm{C}$ to give the "quality of the courses".

\section{Definition 2.4[11]: Fuzzy Matrix}

If a matrix holds an additional property such that all entries belong to closed unit interval $[0,1]$, then it is called Fuzzy Matrix.

\section{Example 2.4}

is a fuzzy matrix of order $4 \times 3$.

\begin{tabular}{|lll}
0.2 & 0.6 & 0.5 \\
0.4 & 0 & 0.9 \\
0.3 & 0.7 & 1 \\
0.1 & 0.2 & 0.8 \\
\hline
\end{tabular}

\section{Definition 2.5[3]: Fuzzy Soft Matrix}

Let $R_{S} \in F S(U)$. Then a fuzzy relation form is defined by

$$
R_{S}=\left\{\left(\mu_{R_{S}}(u, x) /(u, x):(u, x) \in U \times E\right\},\right.
$$

where $\mathrm{U}$ is the universal set, $\mathrm{E}$ is the set of parameters, $\mathrm{S}$ is the subset of $\mathrm{E}$ and the membership function $\mu_{R_{S}}$ is given by

$$
\mu_{R_{S}}: U \times E \rightarrow[0,1] \text { i.e. } \mu_{R_{S}}(u, x)=\mu_{\gamma_{S}(x)}(u) .
$$

If $a_{i j}=\mu_{R_{S}}\left(u_{i}, x_{j}\right)$, for each $\mathrm{i}$ and $\mathrm{j}$, then a fuzzy soft matrix is defined as

$$
\left[a_{i j}\right]_{m \times n}=\left[\begin{array}{cccc}
x_{11} & x_{12} \cdots & x_{1 n} \\
\vdots & \ddots & \vdots \\
x_{m 1} & x_{m 2} \cdots & x_{m n}
\end{array}\right],
$$

and is called $m \times n$ fuzzy soft matrix of the fuzzy set $R_{S}$ over $\mathrm{U}$. 


\section{Definition 2.6[26]: Incomplete Fuzzy Soft Set}

If there are missing values in tabular form of fuzzy soft set, it is called as incomplete fuzzy soft set and unknown values are marked as the sign" * ".

Example2.5. Let $U=\left(u_{i j}\right)_{6 \times 5}$ is an IFSS containing six objects $H=\left(u_{1}, u_{2}, u_{3}, u_{4}, u_{5}, u_{6}\right)$ and five parameters $E=$ $\left(e_{1}, e_{2}, e_{3}, e_{4}, e_{5}\right)$. The matrix representation of the incomplete fuzzy soft set is given in table 2 .

Table 2: Incomplete Fuzzy Soft Matrix

\begin{tabular}{|c|c|c|c|c|c|}
\hline$U / E$ & $\boldsymbol{e}_{\mathbf{1}}$ & $\boldsymbol{e}_{\mathbf{2}}$ & $\boldsymbol{e}_{\mathbf{3}}$ & $\boldsymbol{e}_{\mathbf{4}}$ & $\boldsymbol{e}_{\mathbf{5}}$ \\
\hline $\boldsymbol{u}_{\mathbf{1}}$ & 0.2 & 0.6 & 0.2 & 0.4 & 0.3 \\
\hline $\boldsymbol{u}_{\mathbf{2}}$ & $*$ & 0.4 & 0.3 & 0.3 & 0.6 \\
\hline $\boldsymbol{u}_{\mathbf{3}}$ & 0.7 & $*$ & 0.4 & 0.5 & 0.1 \\
\hline $\boldsymbol{u}_{\mathbf{4}}$ & 0.7 & 0.3 & 0.2 & 0.2 & $*$ \\
\hline $\boldsymbol{u}_{\mathbf{5}}$ & 0.4 & $*$ & 0.3 & $*$ & 0.3 \\
\hline $\boldsymbol{u}_{\mathbf{6}}$ & 0.8 & 0.2 & 0.1 & 0.1 & 0.8 \\
\hline
\end{tabular}

\section{Definition 2.7[11]: Interval Valued Fuzzy Soft Matrix}

An interval valued fuzzy soft matrix of order $m \times n$ is defined as $H=\left(h_{i j}\right)_{m \times n}$, where $h_{i j}=\left[h_{i j}{ }^{-}, h_{i j}{ }^{+}\right]$is $(i, j)^{t h}$ element of $H$ is given as subinterval of $[0,1]$ representing a value of an object corresponding to another object arranged in a rectangular form.

Example2.6. Consider an interval valued fuzzy soft matrix $\mathrm{H}$ given below:

$$
H=\left[\begin{array}{llll}
{[0.5,0.7]} & {[0.3,0.7]} & {[0.1,0.2]} & {[0.1,0.3]} \\
{[0.7,0.9]} & {[0.2,0.6]} & {[0.5,0.9]} & {[0.2,0.7]} \\
{[0.3,0.5]} & {[0.4,0.6]} & {[0.6,0.7]} & {[0.6,0.8]}
\end{array}\right]
$$

\section{ALGORITHM FOR UNKNOWN VALUES ESTIMATION IN INCOMPLETE FUZZY SOFT MATRIX(IFSM)}

Let $U=(H, E)=\left(u_{i j}\right)_{m \times n}, i=1$ to $m, j=1$ to $n$ be an incomplete fuzzy soft set, where $H=\left(h_{1}, h_{2}, \ldots, h_{m}\right)$ be the set of objects and $E=\left(e_{1}, e_{2}, \ldots, e_{n}\right)$ be the set of parameters. The missing entries are denoted by sign ' $*$ ' in matrix representation of $U$. Then, to estimate missing values, the following algorithm is applied:

1. Firstly, calculate mean value $X_{J}$ for each parameter $e_{j} ; j=1$ to $n$ as $X_{j}=\sum_{i=1}^{n} u_{i j} /\left|H_{j}\right|$, where $H_{j}=\left\{i / u_{i j} \neq *, 1 \leq i \leq m\right\}$.

2. Compute average distance denoted by $D_{a v g}\left(X_{j}\right)$ for each entry $u_{i j} ; i=1$ to $m, j=1$ to $n$ as $\left(X_{j}\right)=$ $\sum_{i=1}^{m}\left|X_{j}-u_{i j}\right| /\left|H_{j}\right| \forall j$

where $H_{j}=\left\{i / u_{i j} \neq *, 1 \leq i \leq m\right\}$.

3. Next compute distance deviation $\left\{d_{X_{j}^{+}}, d_{X_{j}^{-}}\right\}$on either side of the mean as

$$
\begin{aligned}
& d_{X_{j}^{+}}=X_{j}+D_{\text {avg }}\left(X_{j}\right) \\
& d_{X_{j}^{-}}=X_{j}-D_{\text {avg }}\left(X_{j}\right) .
\end{aligned}
$$

4. Compare $u_{i j}, j=1$ to $n$ for each $h_{i}, i=1$ to $m$ with mean value $X_{j}, j=1$ to $n, u_{i j} \neq *, 1 \leq i \leq m$. Let $n_{L}$ and $n_{G}$ denotes the number of count for which $u_{i j}<X_{j}$ and $u_{i j} \geq X_{j}$ respectively for corresponding parameter $e_{j}, j=1$ to $n$.

5. By using $n_{L}$ and $n_{G}$ calculate the probabilistic weights $w_{L}$ and $w_{G}$ for each object $h_{i}, i=1$ to $m$ respectively as

$$
w_{L_{i}}=n_{L} /\left(n_{L}+n_{G}\right)
$$




$$
w_{G_{i}}=n_{G} /\left(n_{L}+n_{G}\right) .
$$

6. The missing entries for each parameters $e_{j}$ are computed as

$$
u_{i j}=\left(d_{X_{j}^{+}} \times w_{G_{i}}\right)+\left(d_{X_{j}^{-}} \times w_{L_{i}}\right) \text {, }
$$

where $u_{i j}=*, i=1$ to $m, j=1$ to $n$.

\subsection{Illustration}

Let $U=\left(u_{i j}\right)$ is the FSS containing $U=\left\{h_{1}, h_{2}, h_{3}, h_{4}, h_{5}, h_{6}\right\}$, the set of six houses and the set of parameter is given by $E=\left\{e_{1}=\right.$ expensive, $e_{2}=$ beautiful, $e_{3}=$ wooden, $e_{4}=$ cheap, $e_{5}=$ in green surrounding, $e_{6}=$ modern, $e_{7}=$ in good repair, $e_{8}=$ in bad repair $\}$. Let $\mathrm{Mr} \mathrm{X}$ is interested in buying a house on the basis of his choice of parameters given by $P=\left\{e_{1}, e_{2}, e_{3}, e_{4}, e_{5}\right\}$. The data is arranged in Matrix form as given in table 3 and it found that some of the entries are missing. So, it becomes necessary to estimate these entries before making decision about the buying of house for $\mathrm{Mr} \mathrm{X}$.

The above problem can be written in an Incomplete Fuzzy Soft Matrix as follows:

Table 3: Incomplete Fuzzy Soft Matrix

\begin{tabular}{|c|c|c|c|c|c|}
\hline $\boldsymbol{U} / \boldsymbol{E}$ & $\boldsymbol{e}_{\mathbf{1}}$ & $\boldsymbol{e}_{\mathbf{2}}$ & $\boldsymbol{e}_{\mathbf{3}}$ & $\boldsymbol{e}_{\mathbf{4}}$ & $\boldsymbol{e}_{\mathbf{5}}$ \\
\hline $\boldsymbol{h}_{\mathbf{1}}$ & 0.1 & 0.5 & 0.4 & 0.4 & 0.3 \\
\hline $\boldsymbol{h}_{\mathbf{2}}$ & $*$ & 0.5 & 0.2 & 0.3 & 0.6 \\
\hline $\boldsymbol{h}_{\mathbf{3}}$ & 0.1 & $*$ & 0.3 & 0.5 & 0.1 \\
\hline $\boldsymbol{h}_{\mathbf{4}}$ & 0.7 & 0.2 & 0.2 & 0.2 & 0.3 \\
\hline $\boldsymbol{h}_{\mathbf{5}}$ & 0.2 & 0.5 & 0.3 & $*$ & 0.3 \\
\hline $\boldsymbol{h}_{\mathbf{6}}$ & 0.9 & 0.2 & 0.1 & 0.1 & 0.8 \\
\hline
\end{tabular}

The entries $u_{21}, u_{32}, u_{54}$ are the missing denoted by '*' and are estimated by using algorithm 3.1 as described below:

1. Mean value $X_{1}$ for parameter $e_{1}$ is given by

$X_{1}=(0.1+0.1+0.7+0.2+0.9) / 5=0.4$. Similarly, $X_{2}=0.38, X_{3}=0.25, X_{4}=0.3$ and $X_{5}=0.4$.

2. Average distance $D_{\text {avg }}\left(X_{1}\right)$ is computed as

$D_{\text {avg }}\left(X_{1}\right)=(|0.4-0.1|+|0.4-0.1|+|0.4-0.7|+|0.4-0.2|+|0.4-0.9|) / 5=(0.3+0.3+0.3+0.2+0.5) / 5$

$$
=0.32
$$

Similarly, remaining average distances are $D_{\text {avg }}\left(X_{2}\right)=0.144, D_{\text {avg }}\left(X_{3}\right)=0.1, D_{\text {avg }}\left(X_{4}\right)=0.12$ and $D_{\text {avg }}\left(X_{5}\right)=$ 0.24 .

3. Now, the distance deviations are given below:

$$
\begin{aligned}
& d_{X_{1}^{+}}=0.4+0.32=0.72, d_{X_{1}^{-}}=0.4-0.32=0.08, \\
& d_{X_{2}^{+}}=0.38+0.144=0.524, d_{X_{2}^{-}}=0.38-0.144=0.236, \\
& d_{X_{3}^{+}}=0.25+0.10=0.35, d_{X_{3}^{-}}=0.25-0.10=0.15, \\
& d_{X_{4}^{+}}=0.3+0.12=0.42, d_{X_{4}^{-}}=0.3-0.12=0.18, \\
& d_{X_{5}^{+}}=0.4+0.24=0.64, d_{X_{5}^{-}}=0.4-0.24=0.16 .
\end{aligned}
$$

4. Next $n_{L}$ and $n_{G}$ for corresponding parameter $e_{j}$ are:

In case of object $h_{2}$ for $e_{2}, u_{22}>X_{2}(0.5>0.38)$, for $e_{3}, u_{23}<X_{3}(0.2<0.25)$, for $e_{4}, u_{24}=X_{4}(0.3=0.3)$, for $e_{5}, u_{25}>X_{5}(0.6>0.4)$.

Hence, for $h_{2} n_{L}=1$ and $n_{G}=3$, for object $h_{3}, n_{L}=2, n_{G}=2$ and

for $h_{5}, n_{L}=2, n_{G}=2$.

5. Probabilistic weights $w_{L_{2}}$ and $w_{G_{2}}$ for object $h_{2}$ are given as

$$
w_{L_{2}}=1 /(1+3)=1 / 4, w_{G_{2}}=3 /(1+3)=3 / 4 \text {, }
$$


Thus, for object $h_{3}, w_{L_{3}}=2 /(2+2)=2 / 4, w_{G_{3}}=2 /(2+2)=2 / 4$, and for object $h_{5}, w_{L_{5}}=2 /(2+2)=2 / 4, w_{G_{5}}=2 /(2+2)=2 / 4$.

6. Finally, the missing entries are computed as

$$
\begin{gathered}
u_{21}=(0.72 \times 3 / 4)+(0.8 \times 1 / 4)=0.74, \\
u_{32}=(0.524 \times 1 / 2)+(0.236 \times 1 / 2)=0.38, \\
u_{54}=(0.42 \times 1 / 2)+(0.18 \times 1 / 2)=0.30 .
\end{gathered}
$$

Hence, the missing entries are $u_{21}=0.74, u_{32}=0.38, u_{54}=0.30$.

After substituting the estimated values in table 3, we get fuzzy soft matrix as given below

Table 4: Fuzzy Soft Matrix

\begin{tabular}{|c|c|c|c|c|c|}
\hline $\boldsymbol{U} / \boldsymbol{E}$ & $\boldsymbol{e}_{\mathbf{1}}$ & $\boldsymbol{e}_{\mathbf{2}}$ & $\boldsymbol{e}_{\mathbf{3}}$ & $\boldsymbol{e}_{\mathbf{4}}$ & $\boldsymbol{e}_{\mathbf{5}}$ \\
\hline $\boldsymbol{h}_{\mathbf{1}}$ & 0.1 & 0.5 & 0.4 & 0.4 & 0.3 \\
\hline $\boldsymbol{h}_{\mathbf{2}}$ & $\mathbf{0 . 7 4}$ & 0.5 & 0.2 & 0.3 & 0.6 \\
\hline $\boldsymbol{h}_{\mathbf{3}}$ & 0.1 & $\mathbf{0 . 3 8}$ & 0.3 & 0.5 & 0.1 \\
\hline $\boldsymbol{h}_{\mathbf{4}}$ & 0.7 & 0.2 & 0.2 & 0.2 & 0.3 \\
\hline $\boldsymbol{h}_{\mathbf{5}}$ & 0.2 & 0.5 & 0.3 & $\mathbf{0 . 3 0}$ & 0.3 \\
\hline $\boldsymbol{h}_{\mathbf{6}}$ & 0.9 & 0.2 & 0.1 & 0.1 & 0.8 \\
\hline
\end{tabular}

\section{DIMENSION REDUCTION OF THE DATA}

Here application of FSM in dimension reduction of the data arranged in the table 4 is explained. Firstly, we describe an algorithm for data dimension reduction

\subsection{Algorithm}

The following steps are described which are involve in algorithm for dimension reduction:

1. Input Fuzzy Soft Matrix $(H, E)$.

2. Calculate oriented-object $\operatorname{grade} O_{i}=\left(\sum_{j} u_{i j}\right) /|E|$.

3. Calculate oriented-parameter grade $E_{j}=\left(\sum_{i} u_{i j}\right) /|U|$.

4. Calculate threshold value $T=\left(\sum_{i j} u_{i j}\right) /(|U| \times|E|)$.

5. Remove those rows for which $O_{i}<T$ and those column for which $E_{j}>T$.

6. New matrix is the desire dimensionality reduced matrix.

\subsection{Application of Algorithm}

Considering the fuzzy soft matrix given in Table 4 and applying the algorithm 4.1 , the oriented-object grade $O_{i}$ and oriented-parameter grade $E_{j}$ are calculated as given in Table 5.

Table 5: Oriented Object and Parameter Grades

\begin{tabular}{|c|c|c|c|c|c|l|}
\hline $\boldsymbol{U} / \boldsymbol{E}$ & $\boldsymbol{e}_{\mathbf{1}}$ & $\boldsymbol{e}_{\mathbf{2}}$ & $\boldsymbol{e}_{\mathbf{3}}$ & $\boldsymbol{e}_{\mathbf{4}}$ & $\boldsymbol{e}_{\mathbf{5}}$ & $\boldsymbol{O}_{\boldsymbol{i}}$ \\
\hline $\boldsymbol{h}_{\mathbf{1}}$ & 0.1 & 0.5 & 0.4 & 0.4 & 0.3 & $\mathbf{0 . 3 4}$ \\
\hline $\boldsymbol{h}_{\mathbf{2}}$ & $\mathbf{0 . 7 4}$ & 0.5 & 0.2 & 0.3 & 0.6 & $\mathbf{0 . 4 6}$ \\
\hline $\boldsymbol{h}_{\mathbf{3}}$ & 0.1 & $\mathbf{0 . 3 8}$ & 0.3 & 0.5 & 0.1 & $\mathbf{0 . 2 7 6}$ \\
\hline $\boldsymbol{h}_{\mathbf{4}}$ & 0.7 & 0.2 & 0.2 & 0.2 & 0.3 & $\mathbf{0 . 3 2}$ \\
\hline $\boldsymbol{h}_{\mathbf{5}}$ & 0.2 & 0.5 & 0.3 & $\mathbf{0 . 3 0}$ & 0.3 & $\mathbf{0 . 3 2}$ \\
\hline $\boldsymbol{h}_{\mathbf{6}}$ & 0.9 & 0.2 & 0.1 & 0.1 & 0.8 & $\mathbf{0 . 4 2}$ \\
\hline $\boldsymbol{E}_{\boldsymbol{j}}$ & $\mathbf{0 . 4 5}$ & $\mathbf{0 . 3 8}$ & $\mathbf{0 . 2 5}$ & $\mathbf{0 . 3}$ & $\mathbf{0 . 4}$ & \\
\hline
\end{tabular}


The threshold value $T=\left(\sum_{i j} u_{i j}\right) /(|U| \times|E|)$ is calculated which is 0.356 . Now, remove those rows for which $O_{i}<T$ and those column for which $E_{j}>T$. The reduce matrix is given in table 6 .

Table 6: Reduced Fuzzy Soft Matrix

\begin{tabular}{|c|c|c|c|}
\hline $\boldsymbol{U} / \boldsymbol{E}$ & $\boldsymbol{e}_{\mathbf{3}}$ & $\boldsymbol{e}_{\mathbf{4}}$ & $\boldsymbol{O}_{\boldsymbol{i}}$ \\
\hline $\boldsymbol{h}_{\mathbf{2}}$ & 0.2 & 0.3 & $\mathbf{0 . 4 6}$ \\
\hline $\boldsymbol{h}_{\mathbf{6}}$ & 0.1 & 0.1 & $\mathbf{0 . 4 2}$ \\
\hline $\boldsymbol{E}_{\boldsymbol{j}}$ & $\mathbf{0 . 2 5}$ & $\mathbf{0 . 3}$ & \\
\hline
\end{tabular}

It may be noted that the data size is reduced by $86 \%$ approximately and the maximum object grade value is corresponding to the house $\boldsymbol{h}_{\mathbf{2}}$. Hence, $\mathrm{Mr} \mathrm{X}$ will buy house $\boldsymbol{h}_{\mathbf{2}}$.

\section{ALGORITHM TO ESTIMATE MISSING VALUES IN INCOMPLETE INTERVAL VALUED FUZZY SOFT MATRIX}

In this section an algorithm for estimating missing values in an incomplete IVFSM is proposed and illustrated with an example.

\subsection{Steps of the Algorithm}

Let $U=(H, P)=\left(u_{i j}{ }^{-}, u_{i j}{ }^{+}\right)_{m \times n}, i=1$ to $m, j=1$ to $n$ be an incomplete interval valued fuzzy soft matrix, where $H$ be the set of ' $m$ 'objects and $E$ be the set of ' $n$ 'parameters. Each unknown value $\left(u_{i j}{ }^{-}, u_{i j}{ }^{+}\right)$is denoted by "**'. Then steps of algorithm are given below:

a. Calculate mean value $X_{j}^{-}$and $X_{j}^{+}$for lower and upper membership functions respectively for each parameter $e_{j}$, where, $j=1$ to $n$ as $X_{j}^{-}=\sum_{i=1}^{m} u_{i j}^{-} /\left|H_{i}\right|$ and $X_{j}^{+}=\sum_{i=1}^{m} u_{i j}{ }^{+} /\left|H_{i}\right|$, where $H_{i}=\left\{i / u_{i j}{ }^{-} \neq *, u_{i j}{ }^{+} \neq *, 1 \leq\right.$ $i \leq m\}$.

b. Calculate average distance of $u_{i j}{ }^{-}$and $u_{i j}{ }^{+}$from respective mean $X_{j}^{-}$and $X_{j}{ }^{+}$, denoted by $D_{\text {avg }}\left(X_{j}^{-}\right)$and $D_{\text {avg }}\left(X_{j}^{+}\right)$, where $D_{\text {avg }}\left(X_{j}^{-}\right)=\sum_{i=1}^{m}\left|X_{j}^{-}-u_{i j}{ }^{-}\right| /\left|H_{i}\right|$ and $D_{\text {avg }}\left(X_{j}^{+}\right)=\sum_{i=1}^{m}\left|X_{j}^{+}-u_{i j}^{+}\right| /$ $\left|H_{i}\right|$.

c. Calculate distance deviations $\left\{d_{l} Y_{j}^{-}, d_{l} Y_{j}^{+}\right\}$and $\left\{d_{u} Y_{j}^{-}, d_{u} Y_{j}^{+}\right\}$for lower and upper membership functions respectively from mean $X_{j}^{-}$and $X_{j}^{+}$as $d_{l} Y_{j}^{-}=X_{j}^{-}-D_{a v g}\left(X_{j}^{-}\right), d_{l} Y_{j}^{+}=X_{j}^{-}+D_{a v g}\left(X_{j}^{-}\right)$, and $d_{u} Y_{j}^{-}=$ $X_{j}^{+}-D_{\text {avg }}\left(X_{j}^{+}\right), d_{u} Y_{j}^{+}=X_{j}^{+}+D_{\text {avg }}\left(X_{j}^{+}\right)$.

d. For each object $H_{i}(i=1$ to $m), u_{i j}{ }^{-}$and $u_{i j}{ }^{+}(j=1$ to $n)$ are compared with mean $X_{j}^{-}$and $X_{j}^{+}$respectively $\left(u_{i j}{ }^{-} \neq *, u_{i j}{ }^{+} \neq *\right)$. Let $n_{L}{ }^{-}$and $n_{G}{ }^{-}$be the number of count for which $u_{i j}{ }^{-}<X_{j}^{-}$and $u_{i j}{ }^{-} \geq X_{j}{ }^{-}$for corresponding parameter $e_{j}(j=1$ to $n)$. Similarly, $n_{L}{ }^{+}$and $n_{G}{ }^{+}$be the number of count for which $u_{i j}{ }^{+}<$ $X_{j}^{+}$and $u_{i j}^{+} \geq X_{j}^{+}$, respectively.

e. Using $\left(n_{L}{ }^{-}, n_{G}{ }^{-}\right)$and $\left(n_{L}{ }^{+}, n_{G}{ }^{+}\right)$the probabilistic weight $\left(w_{L_{i}}{ }^{-}, w_{G_{i}}{ }^{-}\right)$and $\left({w_{L}}^{+}, w_{G_{i}}{ }^{+}\right)$of each object $H_{i}(i=1$ to $m)$ are respectively given as $w_{L_{i}}^{-}=n_{L}^{-} /\left(n_{L}^{-}+n_{G}{ }^{-}\right), w_{G_{i}}{ }^{-}=n_{G}{ }^{-} /\left(n_{L}{ }^{-}+n_{G}{ }^{-}\right)$, and $_{L_{i}}{ }^{+}=$ $n_{L}{ }^{+} /\left(n_{L}{ }^{+}+n_{G}{ }^{+}\right), w_{G_{i}}{ }^{+}=n_{G}{ }^{+} /\left(n_{L}{ }^{+}+n_{G}{ }^{+}\right)$.

f. The unknown data for parameter $e_{j}$ is computed as $u_{i j}{ }^{-}=\left(d_{l} Y_{j}^{-} \times{w_{L_{i}}}^{-}\right)+\left(d_{l} Y_{j}^{+} \times w_{G_{i}}{ }^{-}\right)$and $u_{i j}{ }^{+}=$ $\left(d_{u} Y_{j}^{-} \times w_{L_{i}}{ }^{+}\right)+\left(d_{u} Y_{j}^{+} \times w_{G_{i}}{ }^{+}\right)$, where $\left(u_{i j}{ }^{-}\right.$and $\left.u_{i j}{ }^{+}\right)$are unknown entries in IIVFSM $(H, P)$.

\subsection{An Example for Illustration}

Let us consider $R=\left\{h_{1}, h_{2}, h_{3}, h_{4}, h_{5}\right\}$ the set of 5 robots and $P=$ $\left\{e_{1}\right.$ (load capacity), $e_{2}$ (speed), $e_{3}$ (memory capacity), $e_{4}$ (degree of freedom) $\}$ be the set of parameters. An expert evaluates the performance of the robots $h_{i}(i=1$ to 5$)$ with respect to the parameter $e_{j}(j=1$ to 4$)$ and arranged in the form of matrix. But it is found that some of the values are missing. Therefore, before selecting an optimum robot the missing 
data need to be estimated. The data arranged in the form of incomplete matrix is known as incomplete IVFSM as given below in table 7:

Table 7: Incomplete Interval Valued Fuzzy Soft Matrix

\begin{tabular}{|c|c|c|c|c|}
\hline $\boldsymbol{R} / \boldsymbol{P}$ & $\boldsymbol{e}_{\mathbf{1}}$ & $\boldsymbol{e}_{\mathbf{2}}$ & $\boldsymbol{e}_{\mathbf{3}}$ & $\boldsymbol{e}_{\mathbf{4}}$ \\
\hline $\boldsymbol{h}_{\mathbf{1}}$ & {$[0.6,0.8]$} & $*$ & {$[0.5,0.7]$} & {$[0.4,0.5]$} \\
\hline $\boldsymbol{h}_{\mathbf{2}}$ & {$[0.2,0.5]$} & {$[0.7,0.8]$} & {$[0.4,0.8]$} & {$[0.6,0.9]$} \\
\hline $\boldsymbol{h}_{\mathbf{3}}$ & {$[0.5,0.7]$} & {$[0.6,0.8]$} & $*$ & {$[0.8,0.9]$} \\
\hline $\boldsymbol{h}_{\mathbf{4}}$ & {$[0.8,0.9]$} & {$[0.5,0.8]$} & {$[0.8,0.9]$} & {$[0.6,0.7]$} \\
\hline $\boldsymbol{h}_{\mathbf{5}}$ & {$[0.7,0.9]$} & {$[0.6,0.7]$} & $*$ & {$[0.5,0.7]$} \\
\hline
\end{tabular}

Here the values $\left(u_{12}{ }^{-}, u_{12}{ }^{+}\right),\left(u_{33}{ }^{-}, u_{33}{ }^{+}\right)$and $\left(u_{53}{ }^{-}, u_{53}{ }^{+}\right)$are missing, these values are estimated using the algorithm 5.1.

a) Mean values for lower and upper membership function for parameter $e_{1}$ are given by $X_{1}{ }^{-}$and $X_{1}{ }^{+}$respectively as

$X_{1}{ }^{-}=(0.6+0.2+0.5+0.8+0.7) / 5=0.56$ and $X_{1}{ }^{+}=(0.8+0.5+0.7+0.9+0.9) / 5=0.76 . \quad$ Similarly, $\quad$ for parameter $e_{2}, X_{2}^{-}=0.6, X_{2}^{+}=0.78$.

For parameter $e_{3}, X_{3}^{-}=0.57, X_{3}{ }^{+}=0.8$, for $e_{4}, X_{4}^{-}=0.58, X_{4}{ }^{+}=0.74$.

b) Average distances $D_{\text {avg }}\left(X_{1}^{-}\right)$and $D_{\text {avg }}\left(X_{1}^{+}\right)$are calculated as

$$
\begin{gathered}
D_{\text {avg }}\left(X_{1}{ }^{-}\right)=(|0.56-0.6|+|0.56-0.2|+|0.56-0.5|+|0.56-0.8|+|0.56-0.7|) / 5 \\
=(0.04+0.36+0.06+0.24+0.14) / 5=0.17 \\
D_{\text {avg }}\left(X_{1}{ }^{+}\right)=(|0.76-0.8|+|0.76-0.5|+|0.76-0.7|+|0.76-0.9|+|0.76-0.9|) / 5 \\
=(0.04+0.26+0.06+0.14+0.14) / 5=0.13 .
\end{gathered}
$$

Similarly, remaining average distances are given by $D_{\text {avg }}\left(X_{2}^{-}\right)=0.05, D_{\text {avg }}\left(X_{2}{ }^{+}\right)=0.035$,

$D_{\text {avg }}\left(X_{3}^{-}\right)=0.157, D_{\text {avg }}\left(X_{3}^{+}\right)=0.067$ And $D_{\text {avg }}\left(X_{4}^{-}\right)=0.104, D_{\text {avg }}\left(X_{4}^{+}\right)=0.128$.

c) Distance deviations $\left\{d_{l} Y_{1}^{-}, d_{l} Y_{1}^{+}\right\}$and $\left\{d_{u} Y_{1}^{-}, d_{u} Y_{1}^{+}\right\}$for lower and upper membership function from respective mean $X_{1}{ }^{-}$and $X_{1}{ }^{+}$are given below:

$\left\{d_{l} Y_{1}^{-}=|0.56-0.17|=0.39, d_{l} Y_{1}^{+}=|0.56+0.17|=0.73\right\}$ And

Similarly,

$$
\left\{d_{u} Y_{1}^{-}=|0.76-0.13|=0.63, d_{u} Y_{1}^{+}=|0.76+0.13|=0.89\right\} \text {. }
$$

$\left\{\left(d_{l} Y_{2}^{-}=0.55, d_{l} Y_{2}^{+}=0.65\right),\left(d_{u} Y_{2}^{-}=0.75, d_{u} Y_{2}{ }^{+}=0.82\right),\left(d_{l} Y_{3}^{-}=0.41, d_{l} Y_{3}{ }^{+}=0.727\right),\left(d_{u} Y_{3}^{-}=\right.\right.$ $\left.\left.0.73, d_{u} Y_{3}^{+}=0.87\right),\left(d_{l} Y_{4}^{-}=0.48, d_{l} Y_{4}^{+}=0.68\right),\left(d_{u} Y_{4}^{-}=0.61, d_{u} Y_{4}^{+}=0.87\right)\right\}$.

d) $\left(n_{L}{ }^{-}\right.$and $\left.n_{G}{ }^{-}\right)$and $\left(n_{L}{ }^{+}\right.$and $\left.n_{G}{ }^{+}\right)$for each object $H_{i}$ and corresponding to the parameter $e_{j}$ are given as:

For object $H_{1},\left(n_{L}^{-}=2, n_{G}^{-}=1\right)$ and $\left(n_{L}^{+}=2, n_{G}^{+}=1\right)$,

for $H_{2},\left(n_{L}{ }^{-}=2, n_{G}{ }^{-}=2\right)$ and $\left(n_{L}{ }^{+}=1, n_{G}{ }^{+}=3\right)$,

for $H_{3},\left(n_{L}^{-}=1, n_{G}^{-}=2\right)$ and $\left(n_{L}^{+}=1, n_{G}{ }^{+}=2\right)$,

for $H_{4},\left(n_{L}^{-}=1, n_{G}^{-}=3\right)$ and $\left(n_{L}{ }^{+}=1, n_{G}{ }^{+}=3\right)$,

for $H_{5},\left(n_{L}^{-}=1, n_{G}{ }^{-}=2\right)$ and $\left(n_{L}{ }^{+}=2, n_{G}{ }^{+}=1\right)$.

e) Probabilistic weight for object $H_{1}$, is $\left(w_{L_{1}}{ }^{-}=0.67,{w_{G_{1}}}^{-}=0.33\right)$ and $\left({w_{L_{1}}}^{+}=0.67, w_{G_{1}}{ }^{+}=0.33\right)$, for object $H_{2},\left(w_{L_{2}}{ }^{-}=0.5,{w_{G_{2}}}^{-}=0.5\right)$ and $\left({w_{L_{2}}}^{+}=0.25,{w_{G_{2}}}^{+}=0.75\right), \quad$ for $\quad$ object $\quad H_{3},\left(w_{L_{3}}{ }^{-}=0.33, w_{G_{3}}{ }^{-}=\right.$ $0.67)$ and $\left(w_{L_{3}}{ }^{+}=0.33, w_{G_{3}}{ }^{+}=0.67\right)$, for object $H_{4}, \quad\left(w_{L_{4}}{ }^{-}=0.25,{w_{G_{4}}}^{-}=0.75\right)$ and $\left({w_{L}}{ }^{+}=0.25, w_{G_{4}}{ }^{+}=\right.$ 0.75 )and for object $H_{5},\left(w_{L_{5}}{ }^{-}=0.33, w_{G_{5}}{ }^{-}=0.67\right)$ and $\left(w_{L_{5}}{ }^{+}=0.67, w_{G_{5}}{ }^{+}=0.33\right)$.

f) Now the missing entries are given by: $u_{12}{ }^{-}=(0.55 \times 0.67)+(0.65 \times 0.33)=0.37+0.21=0.58, u_{12}{ }^{+}=(0.75 \times$ $0.67)+(0.82 \times 0.33)=0.50+0.27=0.77, u_{33}{ }^{-}=(0.41 \times 0.33)+(0.73 \times 0.67)=0.14+0.49=0.63, u_{33}{ }^{+}=$ $(0.73 \times 0.33)+(0.87 \times 0.67)=0.24+0.58=0.82, u_{53}{ }^{-}=(0.41 \times 0.33)+(0.73 \times 0.67)=0.14+0.49=$ $0.63, u_{53}{ }^{+}=(0.73 \times 0.67)+(0.87 \times 0.33)=0.49+0.29=0.78$.

Therefore, the missing entries are $[0.58,0.77],[0.63,0.82],[0.63,0.78]$. 


\section{MULTI-CRITERIA DECISION MAKING METHOD}

To illustrate the application of interval valued fuzzy soft matrix in decision making, we consider in matrix given in table 8

The steps involve in decision making are given below:

1) Input the interval valued fuzzy soft matrix.

2) Calculate the choice value $c_{i}=\left[c_{i}^{-}, c_{i}{ }^{+}\right]$; where $c_{i}{ }^{-}=\sum_{j=1}^{n} u_{i j}{ }^{-}$and $c_{i}{ }^{+}=\sum_{j=1}^{n} u_{i j}{ }^{+}$.

3) Compute the score value $s_{i}$, given as

$$
s_{i}=\sum_{j=1}^{n}\left\{\left(c_{i}^{-}-c_{j}^{-}\right)+\left(c_{i}^{+}-c_{j}^{+}\right)\right\} .
$$

4) Choose the maximum value of $s_{i}$ that is the requisite decision. If two or more values of $s_{i}$ are same, then choose any one of them according to your wisdom.

Now using the above algorithm decision about the choice of robot given in example 5.2 is taken. After estimating the missing values using algorithm 5.1 the complete IVFSM is given in table 8.

Table 8: Interval Valued Fuzzy Soft Matrix

\begin{tabular}{|c|c|c|c|c|}
\hline $\boldsymbol{R} / \boldsymbol{P}$ & $\boldsymbol{e}_{\mathbf{1}}$ & $\boldsymbol{e}_{\mathbf{2}}$ & $\boldsymbol{e}_{\mathbf{3}}$ & $\boldsymbol{e}_{\mathbf{4}}$ \\
\hline $\boldsymbol{h}_{\mathbf{1}}$ & {$[0.6,0.8]$} & {$[\mathbf{0 . 5 8 , 0 . 7 7}]$} & {$[0.5,0.7]$} & {$[0.4,0.5]$} \\
\hline $\boldsymbol{h}_{\mathbf{2}}$ & {$[0.2,0.5]$} & {$[0.7,0.8]$} & {$[0.4,0.8]$} & {$[0.6,0.9]$} \\
\hline $\boldsymbol{h}_{\mathbf{3}}$ & {$[0.5,0.7]$} & {$[0.6,0.8]$} & {$[\mathbf{0 . 6 3 , 0 . 8 2}]$} & {$[0.8,0.9]$} \\
\hline $\boldsymbol{h}_{\mathbf{4}}$ & {$[0.8,0.9]$} & {$[0.5,0.8]$} & {$[0.8,0.9]$} & {$[0.6,0.7]$} \\
\hline $\boldsymbol{h}_{\mathbf{5}}$ & {$[0.7,0.9]$} & {$[0.6,0.7]$} & {$[\mathbf{0 . 6 3 , 0 . 7 8}]$} & {$[0.5,0.7]$} \\
\hline
\end{tabular}

Compute the choice value $c_{i}=\left[c_{i}{ }^{-}, c_{i}{ }^{+}\right]$as given in table 9 .

Table 9: IVFSM with Choice Value $c_{i}$

\begin{tabular}{|c|c|c|c|c|l|}
\hline $\boldsymbol{R} / \boldsymbol{P}$ & $\boldsymbol{e}_{\mathbf{1}}$ & $\boldsymbol{e}_{\mathbf{2}}$ & $\boldsymbol{e}_{\mathbf{3}}$ & $\boldsymbol{e}_{\mathbf{4}}$ & Choice value $\boldsymbol{c}_{\boldsymbol{i}}$ \\
\hline $\boldsymbol{h}_{\mathbf{1}}$ & {$[0.6,0.8]$} & {$[0.58,0.77]$} & {$[0.5,0.7]$} & {$[0.4,0.5]$} & {$[2.08,2.77]$} \\
\hline $\boldsymbol{h}_{\mathbf{2}}$ & {$[0.2,0.5]$} & {$[0.7,0.8]$} & {$[0.4,0.8]$} & {$[0.6,0.9]$} & {$[1.9,3]$} \\
\hline $\boldsymbol{h}_{\mathbf{3}}$ & {$[0.5,0.7]$} & {$[0.6,0.8]$} & {$[0.63,0.82]$} & {$[0.8,0.9]$} & {$[2.53,3.22]$} \\
\hline $\boldsymbol{h}_{\mathbf{4}}$ & {$[0.8,0.9]$} & {$[0.5,0.8]$} & {$[0.8,0.9]$} & {$[0.6,0.7]$} & {$[2.7,3.3]$} \\
\hline $\boldsymbol{h}_{\mathbf{5}}$ & {$[0.7,0.9]$} & {$[0.6,0.7]$} & {$[0.63,0.78]$} & {$[0.5,0.7]$} & {$[2.43,3.08]$} \\
\hline
\end{tabular}

Now the score value $s_{i}$ is given as

$$
s_{i}=\sum_{j=1}^{n}\left\{\left(c_{i}^{-}-c_{j}^{-}\right)+\left(c_{i}^{+}-c_{j}^{+}\right)\right\} .
$$

Thus, $s_{1}=-2.76, s_{2}=-0.91, s_{3}=1.74, s_{4}=2.99, s_{5}=0.54$. the maximum score value is $s_{4}=2.99$ that is corresponding to the robot 4 . Hence, robot 4 is to be considered for manufacturing purpose.

\section{DISCUSSION AND CONCLUSION}

The estimation of unknown values described in the present paper is very important technique that can be applied in many areas of research, like agriculture, medical diagnosis, economics, and data management. Recently, Hooda and Barak [10] evolved a technique of estimation of missing values in contingency table and illustrated the same. Hooda and Barak [11] 
have also described an interactive technique of missing values estimation in incomplete fuzzy matrix and interval valued fuzzy matrix and have applied the technique in solving a numerical problem.

Surjit Das et al. [7] have also studied an algorithm of estimation of unknown information in incomplete fuzzy soft and interval valued soft sets. In view of the importance and need, we have studied technique to estimate the missing values in incomplete fuzzy soft and interval valued fuzzy soft matrices in the present paper with illustration. The proposed technique makes use of the known information by computing the mean of each parameter and then average distance of each object from the corresponding mean is calculated.

As compared to the approach applied by Deng and Wang [5], our approach is preferably better as we use the weight of probabilities and a pair of deviations to retrieve the missing data. We have also studied the application of incomplete fuzzy soft and interval valued fuzzy soft matrices in decision making problem after estimating the unknown values in data.

Conflict of Address: It is stated that there is no conflict of interest among authors.

\section{REFERENCES}

[1] Chen D., Tsang E.C.C., Yeung D.S. and Wang X. The Parameterization Reduction of Soft Sets and its Application, Computer and Mathematics with Applications, 49(2005), 757-763.

[2] Cagman N., Enginoglu S. Soft Matrices and its Decision Making, Computer and Mathematics with Applications, 59(2010), 3308-3314.

[3] Cagman N., and Enginoglo S. Fuzzy Soft Matrix Theory and its Application in Decision Making, International Journal of Fuzzy Systems, 9(2012), 109-119.

[4] Celik Y. and Yamak S. Fuzzy Soft Set Theory Applied to Medical Diagnosis using Fuzzy Arithmetic Operations, Journal of Inequalities and Applications, DOI: https://doi.org/10.1186/1029-242x-2013.

[5] Deng T. and Wang X. An Object-Parameter Approach to Predicting Unknown Data in Incomplete Fuzzy Soft Sets, Applied Mathematical Modelling,37(2013), 4139-4146.

[6] Dong Y. and Xiao Z. A Group Decision Making Method Based on Dempster-Shafer Fuzzy Soft Sets under Incomplete Information, International Journal of Hybrid Information Technology, 8(2015), 287-296.

[7] Das S., Ghosh S. and Pal T. An Algorithmic Approach for Predicting Unknown Information in Incomplete Fuzzy Soft Set, Arabian Journal for Sciences and Engineering, 42(2017), 3563-3571.

[8] Hooda D.S. and Jain D. Measures of Information on Fuzzy Soft Matrix and Fuzzy Binary Relation, Journal of Combinatories, Information and System Sciences, 37(2012), 1-18.

[9] Hooda D.S. and Kumari R. On Application of Fuzzy Soft Set in Dimension Reduction and Medical Diagnosis, Advance in research, 12(2017), 1-9.

[10] Hooda D.S. and Barak M.S. Estimation of Missing Data in Design of Experiment and Contingency Table, $S N$ Applied Sciences, DOI: https://doi.org/10.1007/s42452-019-0692-0, 2019.

[11] Hooda D.S. and Barak M.S. Estimation of Missing Values in Fuzzy Matrix and Interval Valued Fuzzy Matrix, Life Cycle of Reliability and Safety Engineering, DOI: https://doi.org/10.1007/s41872-020-00116, 2020.

[12] Jun Y.B. and Song S.Z. Soft Sub-algebra and Soft Ideals of BCK/BCI- Algebras Related to Fuzzy Set Theory, Mathematical Communication, 14(2009), 271-281.

[13] Kandasamy W.B.V., Smarandache F. and Kandasamy L. Fuzzy Matrix Entropy Theory and Fuzzy Models for Social Scientists, Automation Los Angles, USA, DOI: 10.6084/M9.FIGSHARE.101549/.2007.

[14] Kong Z., Gao L. and Wang L.S. The Normal Parameter Reduction of Soft Set and its Algorithm, Computer and Mathematics with Application, 56(2008), 3029-3037.

[15] Kong Z.,Gao L. and Wang L.S. Comment on "A Fuzzy Soft Set Theoretic Approach to Decision Making Problems", Journal ofComputational and Applied Mathematics,223(2009), 540-542.

[16] Kalayathankal S.J. and Singh S. A fuzzy Soft Flood Alarm Model, Mathematics and Computers in Simulation, 80(2010), 887-893.

[17] Kalaichelvi A. and Malini P.H. Applications of Fuzzy Soft Sets to Investment Decision Making Problems, International Journal of Mathematical Sciences and Applications, 3(2011), 1583-1586.

[18] Khan M.S., Algaradi M.A, Abdul Wahab A.W. and Herawan T. An Alternative Data Filling Approach for Predicting of Missing Data in Soft Sets (ADFIS), Springer Plus, 5(2016), 1348.

[19] Molodtsov D.A. Soft Set Theory- First Result, Computers and Mathematics with Applications, 37(1999), 1931.

[20] Maji P.K., Biswas R. and Roy A.R. Fuzzy Soft Sets, Journal of Fuzzy Mathematics, 9(2001), 589-602. 
[21] Maji P.K., Biswas R. and Roy A.R. An Application of Soft Set in Decision Making Problem, Computer and Mathematics with Application, 44(2002), 1077-1083.

[22] Maji P.K., Roy A.R. and Biswas R. On Intuitionistic Fuzzy Soft Set, Journal of Fuzzy Mathematics, 12(2004), 669-683.

[23] Ma X., Sulaiman N., Qin H., Herawan T. and Zain J.M. A New Efficient Normal Parameter Reduction Algorithm of Soft Sets, Computer and Mathematics with Applications, 62(2011), 599-598.

[24] Qin H., Ma X., Herawan T. and Zain J.M. Data Filling Approach of Soft Sets under Incomplete Information, In: Nguyen, N.T., Kim, C. G. Janiak, A. (eds) ACIIDS 2011, LNAI, 6592(2011), 302-311.

[25] Tanay B. and Kandemir M.B. Topological Structure of Fuzzy Soft Set, Computer and Mathematics with Application, 61(2011), 2952-2957.

[26] Wang L. and Qin K. Incomplete Fuzzy Soft Set and their Application to Decision Making. DOI: 103390/Sym11040535.

[27] Zadeh L A. Fuzzy sets. Information and Control, 8(1965), 338-353.

[28] Zou Y. and Xiao Z. Data Analysis Approach of Soft Sets under Incomplete Information, Knowledge Based System, 21(2008), 941-945. 